\title{
Fatal Multi-Focal Necrotizing Soft Tissue Infection in an Immunocompromised Patient
}

\author{
Adam C. Sheka and Michele Loor
}

\begin{abstract}
Background: Necrotizing soft tissue infection (NSTI) is an uncommon but devastating disease process. Multifocal NSTI is even more rare. Atypical presentations of NSTI delay definitive treatment with aggressive surgical debridement.

Case Presentation: A 26-year-old Asian female who had undergone recent chemotherapy presented to the emergency department with vaginal bleeding. She was admitted for observation and developed rapidly progressive bilateral leg pain and ecchymosis. After the development of crepitus, the surgery team was consulted. Because of high suspicion for NSTI, she was taken to the operating room for surgical debridement. She was found to have extensive myonecrosis in her bilateral lower extremities necessitating amputations of both legs. Her left forearm was also explored, with all three extremities found to harbor gram-positive bacilli. Despite aggressive debridement and maximal intensive cares, the patient died on her first post-operative day because of Clostridium perfringens sepsis.

Conclusion: Providers who care for immunocompromised patients must have a lower threshold to consider NSTI in their differential diagnosis. Immunocompromised patients may present in an atypical fashion, which delays diagnosis and treatment.
\end{abstract}

Keywords: necrotizing fasciitis; necrotizing soft tissue infection; Clostridium perfringens

$\mathbf{N}$ ECROTIZING SOFT TISSUE INFECTIONS (NSTI) are an uncommon but devastating disease entity resulting in widespread necrosis of fascia, subcutaneous tissue, and muscle if not promptly treated with aggressive surgical debridement. Early diagnosis and operative intervention are essential for survival and meaningful recovery. Although rare, with an estimated four cases per 100,000 person-years in the United States, mortality rates of $25 \%-35 \%$ or higher are reported [1]. Immunocompromised persons are at higher risk for NSTIs and typically have a higher mortality [2].

Multi-focal involvement of non-contiguous sites is even more rare but has been published in multiple case series and individual reports [3-5]. Retrospective analyses indicate that multifocal NSTIs have a higher mortality rate than infections of a single site [1].

Here, we present an atypical case of multifocal NSTI in an immunocompromised patient resulting in widespread Clostridium myonecrosis with a fatal outcome despite aggressive surgical debridement.

\section{Case Presentation}

A 26-year-old Asian female was brought to the emergency department with two days of vaginal bleeding, weakness, fatigue, and cramping abdominal pain. Her past medical history was notable for acute myeloid leukemia, for which she underwent induction chemotherapy with cytarabine and daunorubicin; she had completed her fourth cycle of highdose cytarabine seven days prior to presentation. She was hypotensive on arrival and pancytopenic with hemoglobin of $6.9 \mathrm{~g} / \mathrm{dL}$, platelet count of 23,000 cells $/ \mathrm{mm}^{3}$, and white blood cell count of less than 100 cells $/ \mathrm{mm}^{3}$. She was given one unit of platelets and one unit of packed red blood cells. A speculum examination and a pelvic ultrasound were performed in the emergency department and did not show any abnormalities. She was admitted for observation.

Around 11:00 PM, approximately $16 \mathrm{~h}$ after presentation, the patient began to complain of bilateral calf pain. Shortly thereafter, she became tachycardic with heart rates in the

Department of Surgery, University of Minnesota, Minneapolis, Minnesota.

(C) Adam C. Sheka and Michele Loor. 2017; Published by Mary Ann Liebert, Inc. This Open Access article is distributed under the terms of the Creative Commons License (http://creativecommons.org/licenses/by/4.0), which permits unrestricted use, distribution, and reproduction in any medium, provided the original work is properly credited. 
$120 \mathrm{~s}$ and developed a fever (temperature, $102.7^{\circ} \mathrm{F}$ ). A serum lactate was drawn and found to be $4.4 \mathrm{mmol} / \mathrm{L}$. A transfusion reaction rule-out was initiated with the blood bank. She was also started on prophylactic vancomycin and cefepime for febrile neutropenia. Around 5:30 AM, she had worsening calf pain, as well as tingling in the right foot. Her tachycardia worsened to the 160 s despite resuscitation. An area of dark purple ecchymosis was noted on her right calf, which was becoming increasingly taut and tender. The general surgery team was consulted because of concern for calf hematoma with compartment syndrome.

When she was observed by the surgery team, she was toxic in appearance, tachycardic with a heart rate of 155 and febrile to $102.5^{\circ} \mathrm{F}$. The area of ecchymosis over her right calf extended beyond the previously marked boundaries and a new area of ecchymosis had appeared on her left leg. She exhibited exquisite tenderness to touch on both calves and extreme pain with passive extension and flexion of her ankles. She had easily palpable dorsalis pedis and posterior tibial pulses bilaterally, although sensation was diminished in her right foot. Crepitus was noted at her right ankle.

Because of high suspicion for NSTI, she was brought to the operating room for emergent incision and debridement. Additional antibiotic coverage with piperacillin-tazobactam and clindamycin were initiated. She was in the operating room within one hour of being observed by the surgery team. The ecchymosis had continued to spread and crepitus was palpable to the level of the knee on her right lower extremity by the time incision was made (Fig. 1). As she was being draped for the operation, an additional area of ecchymosis was noted on her left forearm.

An incision was first made over her right medial calf. There was immediate extravasation of air from each fascial compartment entered. Extensive myonecrosis was found extending to the level of the distal thigh, where viable muscle tissue remained that was reactive to electrocautery. During this exploration, the patient decompensated and became hypotensive, coagulopathic, and acidotic. A hemoglobin was drawn and found to be $4.0 \mathrm{~g} / \mathrm{dL}$. Massive transfusion protocol was initiated. Once the patient was stabilized, the left leg was explored in a similar fashion with myonecrosis found to the level of the proximal calf. Intraoperative gram stain from both lower extremities returned gram-positive bacilli. At this point, bilateral amputations were discussed with the family and an exploration of the left forearm was performed, which found gross air in the volar compartment but viable tissue. A gram stain similarly returned gram-positive bacilli. Right above-knee and left below-knee guillotine amputations were performed. The left forearm was packed and remained open. The patient was taken to the surgical intensive care unit post-operatively where despite maximal therapy, she continued to decompensate overnight. Ecchymoses spread proximally from her bilateral lower extremities and her left forearm. The family elected to transition to comfort care. The patient died on the first post-operative day. Final tissue cultures grew Clostridium perfringens.

\section{Discussion}

Clostridial infections seem the most likely bacterial culprit for historical descriptions of NSTI, such as the "gas gangrene" described during the era of the the American Civil War. In contemporary series of NSTI, Clostridium species are isolated less commonly, likely because of public health improvements, but they still represent a sizable portion of NSTIs in areas in which these species are endemic [2]. The gram-positive, rod-shaped, anaerobic, spore-forming bacteria are common in nature, present in soil, marine sediment, and as normal flora in the human digestive tract. Clostridium perfringens has also been reported as a part of normal vaginal flora in $3 \%-10 \%$ of healthy women [6]. The patient's pelvic ultrasound is a possible source for her infection. Studies suggest only $5 \%$ of Clostridium strains are pathologically virulent, [6] however, these infections progress rapidly and once a patient demonstrates shock, mortality exceeds $50 \%$ $[2,7]$. The overall mortality rate of Clostridium sepsis is even higher when infection induces intense hemolysis, the most notable negative prognostic indicator associated with the

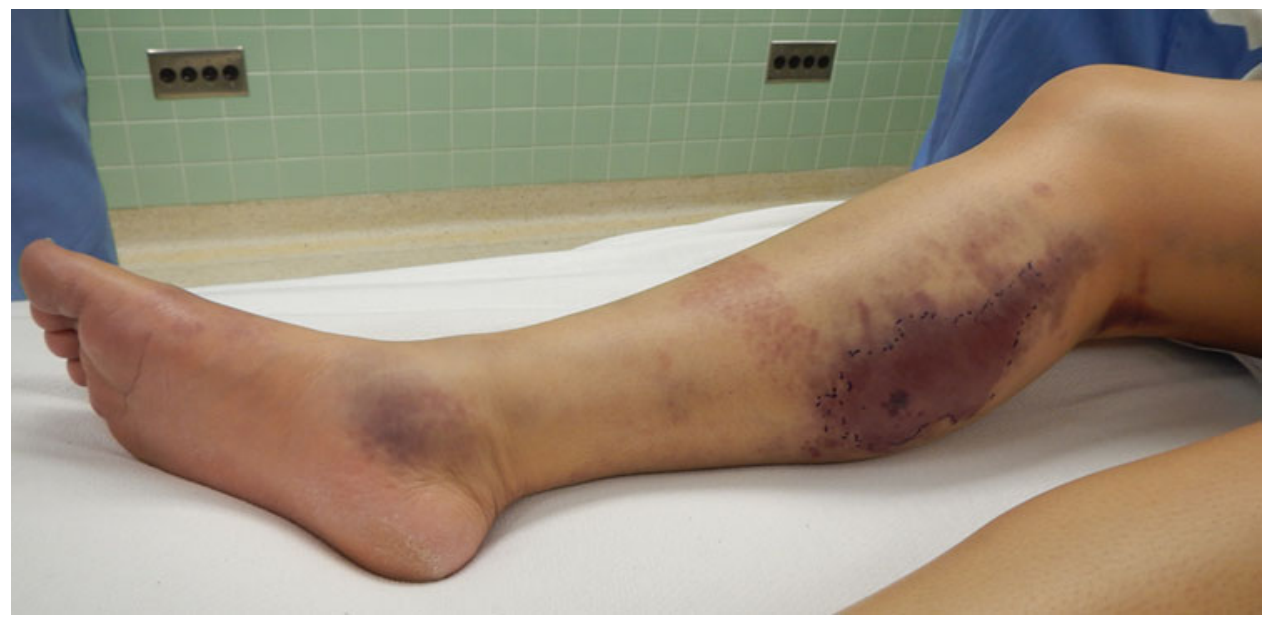

FIG. 1. Right lower extremity immediately prior to incision. Note advancement of ecchymosis beyond previously demarcated area. 
infection [7]. The virulence of Clostridium is dependent on the presence of a factor called $\alpha$-toxin. Early in the infection, $\alpha$-toxin acts as a potent platelet agonist, resulting in formation of thrombi with the resultant local tissue ischemia establishing favorable growth conditions for the bacteria. Later in the course, as it spreads systemically, $\alpha$-toxin acts to hemolyze red blood cell membranes directly [2,7]. Our patient demonstrated several hallmarks of Clostridium sepsis, including rapid progression of her disease process, palpable crepitus on physical examination, gross extravasation of gas from her fascial compartments, and hemolytic anemia requiring massive intra-operative transfusion.

Necrotizing soft tissue infection in immunocompromised patients presents a diagnostic challenge. Laboratory evaluations typically predictive of NSTI are no longer useful. The most commonly used tool for predicting NSTI is the laboratory risk indicator for necrotizing fasciitis score (LRINEC) [8]. The tool assigns scores based on laboratory values for C-reactive protein, white count, hemoglobin, sodium, creatinine, and glucose concentrations (Table 1). However, this scoring system pre-supposes a competent immune reaction to the infection. In our patient, the C-reactive protein, white count, sodium, creatinine, and glucose were below positive concentrations for the LRINEC system. Only hemoglobin would would have generated points on the scale, with hemoglobin less than $11 \mathrm{~g} / \mathrm{dL}$ adding two points. A score less than five indicates a low risk of necrotizing soft tissue infection, or less than $50 \%$ of the time.

TABLE 1. LABORATORY RISK INDICATOR FOR NeCROTIZING FASCIITIS (LRINEC) ScORE

\begin{tabular}{lc}
\hline Laboratory value & Score \\
\hline C-reactive protein $(\mathrm{mg} / \mathrm{L})$ & \\
$\quad<150$ & 0 \\
$\quad \geq 150$ & 4 \\
Total white cell count $\left(\right.$ cells $\left./ \mathrm{mm}^{3}\right)$ & \\
$\quad<15$ & 0 \\
$\quad 15-25$ & 1 \\
$\quad>25$ & 2 \\
Hemoglobin $(\mathrm{g} / \mathrm{dL})$ & \\
$\quad>13.5$ & 0 \\
$\quad 11-13.5$ & 1 \\
$\quad<11$ & 2 \\
Sodium $(\mathrm{mmol} / \mathrm{L})$ & \\
$\quad \geq 135$ & 0 \\
$\quad<135$ & 2 \\
Creatinine $(\mathrm{mg} / \mathrm{dL})$ & \\
$\quad \leq 1.6$ & 0 \\
$\quad>1.6$ & 2 \\
Glucose $(\mathrm{mg} / \mathrm{dL})$ & \\
$\quad \leq 180$ & 0 \\
$>180$ & 1
\end{tabular}

Total Score

$\leq 5$, low, $<50 \%$ risk

$6-7$, moderate, $50 \%-75 \%$ risk

$\geq 8$, high, $>75 \%$ risk
Patients with immune compromise are at higher risk for NSTI and do not present in a typical fashion. Thus, providers who care for immunocompromised patients must have a lower threshold to place NSTI on their differential diagnosis. A study utilizing the National Surgical Quality Improvement Program registry found that patients with NSTI were twice as likely as controls to be taking steroid medications. The same study found that NSTI patients with a cancer diagnosis had a five-fold increased mortality risk [9]. Another retrospective study found that immunocompromised patients with NSTI had double the in-hospital mortality of immunocompetent patients and were less likely to have an elevated white count, be admitted to a surgical service, or undergo surgical debridement on admission. In addition, time to diagnosis and first surgical debridement were delayed in immunocompromised patients [10]. This delay is clear in the case of our patient, in whom a diagnosis of febrile neutropenia was adopted and treated despite a specific, focal complaint of leg pain.

Our patient's initial complaint related to NSTI was bilateral leg pain, another atypical presentation, as most cases are confined to a single limb or contiguous area. The literature suggests $5 \%-34 \%$ of NSTI cases have synchronous, multifocal involvement. Compared with patients with single sites of infection, those with multi-focal NSTI have higher inhospital mortality, with rates up to $67 \%$ reported [3-5], likely caused by delays in diagnosis and treatment.

\section{Conclusion}

Necrotizing soft tissue infections are a rare but devastating disease process requiring prompt diagnosis and surgical debridement. Immunocompromised patients are at higher risk for NSTI and have higher mortality. Thus, providers who care for immunocompromised patients must have a lower threshold to consider NSTI in their differential alongside more common diagnoses such as febrile neutropenia. The potential for multi-focal infection is another confounding factor that providers must contemplate.

\section{Author Disclosure Statement}

No competing financial interests exist.

\section{References}

1. Hua C, Sbidian E, Hemery F, et al. Prognostic factors in necrotizing soft-tissue infections (NSTI): A cohort study. J Am Acad Dermatol 2015;73:1006-1012.

2. Hakkarainen TW, Kopari NM, Pham TN, et al. Necrotizing soft tissue infections: Review and current concepts in treatment, systems of care, and outcomes. Curr Probl Surg 2014; 51:344-362.

3. Lee $\mathrm{C}, \mathrm{Li} \mathrm{Y}$, Huang $\mathrm{T}$, et al. Synchronous multifocal necrotizing fasciitis prognostic factors: A retrospective case series study in a single center. Infection 2016;44: 757-763.

4. El-Khani U, Nehme J, Darwish A, et al. Multifocal necrotising fasciitis: An overlooked entity? J Plast Reconstr Anesthet Surg 2012;65:501-512. 
Address correspondence to: Dr. Adam C. Sheka

I, Lancerotto L, Pontini A, et al "Synchronous" multifocal necrotizing fasciitis. J Emer Med 2013;45:e187-e191.

6. Hendrix NW, Mackeen AD, Weiner S. Clostridium perfringens sepsis and fetal demise after genetic amniocentesis. Am J Perinatol Rep 2011;1:25-28.

7. van Bunderen CC, Bomers MK, Wesdorp E, et al. Clostridium perfringens septicaemia with massive intravascular haemolysis: A case report and review of the literature. Neth J Med 2010;68:343-346.

8. Wong C, Khin L, Heng K, et al. The LRINEC (laboratory risk indicator for necrotizing fasciitis) score: A tool for distinguishing necrotizing fasciitis from other soft tissue infections. Crit Care Med 2004;32:1535-1541.

9. Mills MK, Faraklas I, Davis C, et al. Outcomes from treatment of necrotizing soft-tissue infections: Results from the National Surgical Quality Improvement Program database. Am J Surg 2010;200:790-797.

10. Keung EZ, Liu X, Nuzhad A, et al. Immunocompromised status in patients with necrotizing soft-tissue infection. JAMA Surg 2013;148:419-426.
Department of Surgery

MMC 195

420 Delaware Street $S E$ Minneapolis, MN 55455

E-mail: sheka015@umn.edu

Abbreviation Used
$\mathrm{NSTI}=$ necrotizing soft tissue infection

Cite this article as: Sheka AC, Loor M. Fatal multifocal necrotizing soft tissue infection in an immunocompromised patient. Surgical Infections Case Reports 2:1, 65-68, DOI: 10.1089/crsi.2017.0014 University of Minnesota 